\title{
Kannada Handwritten numeral Recognition using FFBPNN Classifiers
}

\author{
Ashoka H N \\ Department of Electronics and \\ Communication Engineering, \\ Sikkim Manipal University, Sikkim \\ India.
}

\author{
Manjaiah D H \\ Department of Computer Science \\ Manglore university,Mangalore, \\ India
}

\author{
Rabindranath Bera \\ Department of Electronics and \\ Communication Engineering, \\ Sikkim Manipal University, Sikkim \\ India
}

\begin{abstract}
This paper presents the performance of Kannada handwritten numeral recognition using feed forward back propagation neural network (FFBPNN) classifiers. The classifier is designed to recognize the Kannada handwritten numerals. Samples are represented by the few features extracted by the zoning technique. The input numeral samples in binary form are stored in a fixed window size of $12 \times 12$ and partitioned into nine sub regions of $4 \mathrm{x} 4$ sizes for their representation. A normalized feature value is computed by the one's present each sub region for their representation in two different approaches. On experimentation, it is found the overall recognition rate of $99.7 \%$ and $95.5 \%$ for the feature extraction approaches $\mathrm{M}_{1}$ and $\mathrm{M}_{2}$ respectively.
\end{abstract}

\section{General Terms}

Pattern Recognition, Feed Forward Back Propagation Neural Network, Character Recognition

\section{Keywords}

Feature extraction, Knowledge base, Kannada handwritten numeral recognition

\section{INTRODUCTION}

The Kannada is the popular language of south Indian state Karnataka. It has separate numeral symbols and used extensively to write the postal code, bank cheques, vehicle and street numbers. Due to their numerous applications in the state, automated reading and recognition of handwritten Kannada numerals finds intensive research this decade. From the past five decades, various techniques have been proposed for the off-line character recognition system and they generally differ in the feature extraction, feature selection and classification techniques. The classification and recognition efficiency depends on these processes.

Pattern representation plays an important role in the design of classifier for character recognition. The representation is identifying the various characteristic attributes or the relevant information associated in the pattern. This process is called feature extraction in pattern classification and recognition. The statistical, global transformation and series expansion, and geometrical and topological techniques are the commonly used feature extraction methods in the handwritten character representation $[1,2]$. The features extracted for character representation need to have minimum variation in the intraclass and maximum variation among the interclass. The features such extracted needs to be an independent of the size, orientation to overcome the variations involved in the writing style [3]. The zoning technique of statistical method is extensively used for feature extraction in Indian language handwritten numeral recognition [4]. In this technique, the scanned images of numerals are transformed to a binary form and divided into a number of overlapping and nonoverlapping zones and the density of $1 \mathrm{~s}$ in each zone transformed into a feature. Although in this type of representation, the reconstruction of the original image is not possible but it reduces the dimension of pattern.

Hanumandlu and Murthy [5] divided each character image into 24 zones and considering the bottom left corner as the absolute origin, they computed coordinate vector distance of $1 \mathrm{~s}$ pixel for the zone feature. The feature vectors such extracted are then used to construct the fuzzy classifier. They reported a overall recognition rate of $95 \%$ on Hindi and 98.4\% on English numeral database. O P Sharma et al [6] extracted in total 69 features for alphabet representation by the zoning technique. Features such extracted are used to train the FFBPNN for performing classification and recognition tasks and reported the accuracy up to $98.5 \%$.

Rajashekaradhya and Ranjan [7] extracted two features from each of the 50 zones for four popular south Indian scripts handwritten numerals recognition. The nearest neighbor and the feed forward back propagation neural network classifiers are used for classification and recognition purpose. They reported the recognition rate of $99 \%, 99 \%, 96 \%$ and $95 \%$ for Kannada, Telugu, Tamil and Malayalam numerals respectively. Dinesh Acharya et.al [8] extracted 44 potential different types of structural features for the recognition of isolated handwritten Kannada numerals and reported a recognition accuracy of $98 \%$ from the fuzzy k-NN classifiers. Dhanrda et.al [9] divided the binary image of a digit into 64 zones and computed feature for each zone by the pixel density for bilingual Kannada and Telugu digits recognition. They reported a recognition rate of $95.5 \%$ and $99.83 \%$ with K-NN classifier for Kannada and Telugu numerals respectively.

U. Pala et al [10] extracted features from the angular external and internal contour pixels information of the characters for the recognition. The Circular and convex hull rings have been used to divide the character into smaller zones to get the zonewise features for higher recognition results. They combined circular and convex hull features to improve the results and these features are fed to support vector machines (SVM) for recognition and reported recognition rate of $99.18 \%$ on Devnagari and $98.86 \%$ on Bangla character.

In this view, an attempt was made to construct the classifier for Hindu-Arabic numeral recognition using nine features in the earlier work [11]. The work presented in this paper also extracts nine features for Kannada handwritten numerals representation by the zoning feature extraction technique and used train the classifier. 


\section{METHODOLOGY}

The methodology of the work presented in this paper is shown diagrammatically in the Fig.1. The handwritten Kannada numeral database in binary form is divided into training and testing data. The training database is used to construct the knowledge base and the testing data to evaluate the performance. The binary image of a sample is partitioned into number of sub-regions to extract a features used to train the FFBNN.

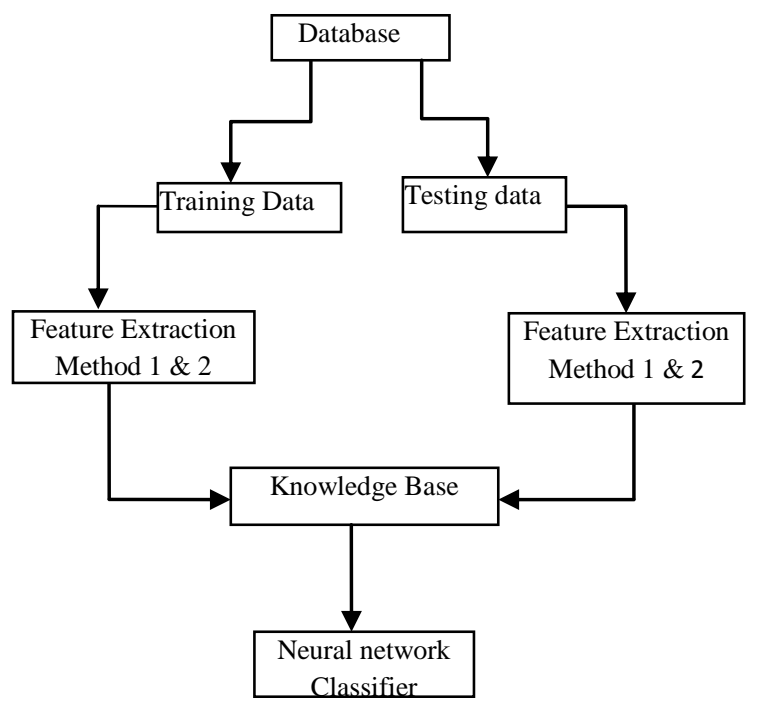

Fig.1. Methodology used for the recognition system

\section{DATABASE}

As the standard Kannada handwritten numeral database not available for the experimentation [12], the same database constructed for the earlier work [13] is used in this work also. The database was collected from 125 different age group writers. Each writer was provided two A4 sheets having ten squares in a row with the $8 \mathrm{~mm} \times 8 \mathrm{~mm}$ in size. Each writer was requested to write 0-9 Kannada numerals one time on both the sheets and used one sheet for the training data and another for the testing data samples. These data sheets are scanned using HP-scan jet 5400c at 300 dpi. The darkness introduced in the background during the scan is reduced slightly by increasing the brightness of image. The numeral images in the scanned sheets are segmented and stored in JPEG format separately. These numeral images are binarized and fixed in a window of $12 \times 12$ size by the MATLAB builtin functions. In this work, to train the FFBPNN, only first 22 samples of each numeral class are selected. In total, the training set consist 220 samples from the ten numeral classes.

\section{FEATURE EXTRACTION}

The steps involved in feature extraction process are given in the Fig.2. In the process, the input samples binary images are partitioned into a nine square regions of $4 \times 4$ sizes and computed a real number from each region for their representation. A feature value of each region is computed by the number of one's present in it by the two different approaches $M_{1}$ and $M_{2}$ [11]. The feature sets such extracted are used to train the network and evaluated the performance separately.

In the first approach $\mathrm{M}_{1}$, a feature value is computed by dividing the total number of one's elements in a region from the total number of elements in that region. The feature value, $f_{r}$ of $r^{\text {th }}$ region is given in the Eq. (1).

$$
f_{r}=\frac{1}{q_{r}} \sum_{i=1}^{q_{r}} p_{i j} j=1,2 \ldots \ldots . n
$$

Where, $f_{r}=$ feature value of $\mathrm{r}^{\text {th }}$ region, $q_{r}=$ total number of cells in the region $\mathrm{r}, P_{i j}=$ value of cell in $\mathrm{i}^{\text {th }}$ row $\mathrm{j}^{\text {th }}$ column of a matrix $\mathrm{p}$ in the region $\mathrm{r}$.

In the second approach $\mathrm{M}_{2}$, the feature value is computed by dividing the sum coordinate distance of one's elements from the sum coordinate vector distances of all the elements in the region. The coordinate distance of each element, $d_{i j}^{r}$ of $i^{\text {th }}$ element in $\mathrm{r}^{\text {th }}$ region, is measured by the Eq. (2) and the normalized feature value $f_{r}$ for the region ' $r$ ' is computed using the Eq. (3).

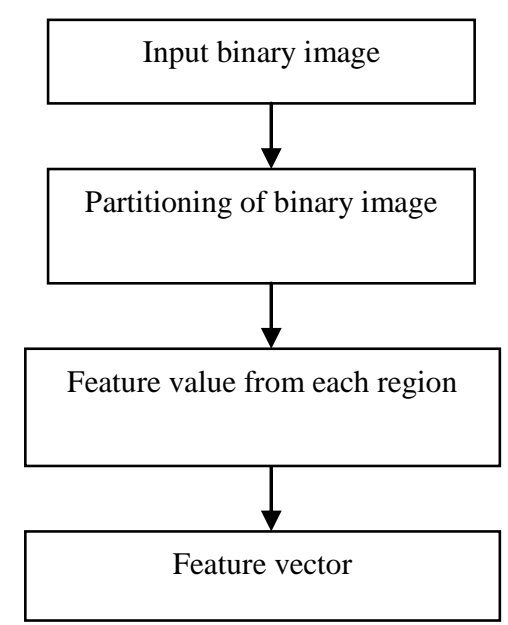

Fig. 2. The steps of feature extraction process

$$
\begin{array}{r}
\mathrm{d}_{\mathrm{ij}}^{\mathrm{r}}=\sqrt{\mathrm{i}^{2}+\mathrm{j}^{2}} \\
f_{r}=\frac{\sum_{i, P_{i j}=1}^{q_{r}} d_{i j}^{r}}{\sum_{i}^{q_{r}} d_{i j}^{r}}
\end{array}
$$

where, $d_{i j}^{r}$ is the $r^{\text {th }}$ region coordinate vector distance of $i^{\text {th }}$ row $j^{\text {th }}$ column of matrix, $f_{r}=r^{\text {th }}$ region feature value, $\mathrm{q}_{\mathrm{r}}=$ total number of elements in $\mathrm{r}^{\text {th }}$ region.

The features such extracted to represent a sample are stored together and is called the feature vector. These feature vectors consists normalized values and are directly used to train the back propagation neural network classifiers.

\section{CLASSIFICATION AND RECOGNITION}

After extracting the feature vectors to represent the samples in the classification procedure, classifiers are designed using them. In practice, the classifier choice is based on the available or best known to the designer. In this work, the extensively used FFBPNN classifier in pattern recognition is used and it is designed to estimate the correct class of unknown sample.

The designed FFBPNN for Kannada numeral recognition has three (input, hidden, and output) layers. The nodes in input 
layer are made equal to the number of features extracted to represent the character and they made equal to database classes in the output layer. There are different methods to decide the number of nodes in hidden layer and is out scope of this and our work. Presently, taken the number of nodes in hidden layer are less than twice the input layer and more than the output layer. The designed FFBPNN has 9-12-10 nodes in the input, hidden and output layers respectively.

The initial weight matrixes of the FFBPNN are set randomly and used sigmoid activation function in both the input and output layers. The input-output relations of training set samples are shown one after the other to the FFBPNN and allowed to learning. The neural network is made to learn with different learning rate ' $\alpha$ '. The Root Mean Square Error (RMSE) is used as the performance indicator to terminate the learning process. The weight matrixes of hidden and output layers at the end of FFBPNN learning is then used to recognize the unknown samples.

\section{RESULTS AND DISCUSSIONS}

The FFBPNN classifier is trained by the set of features extracted by the approaches $\mathrm{M}_{1}$ and $\mathrm{M}_{2}$ separately. The Kannada handwritten numeral recognition results and the performance on two feature extraction methods are presented in this section.

The feature vectors for each of the samples are extracted by the approaches $\mathrm{M}_{1}$ and $\mathrm{M}_{2}$. To know the rigidity towards writing style variation of the feature extraction approaches, the variation among the intraclass regions features is observed. Fig. 3 and Fig. 4 shows the feature vectors of all the 22 training samples of numeral class ' 0 ' extracted by the approach $\mathrm{M}_{1}$ and $\mathrm{M}_{2}$ respectively.

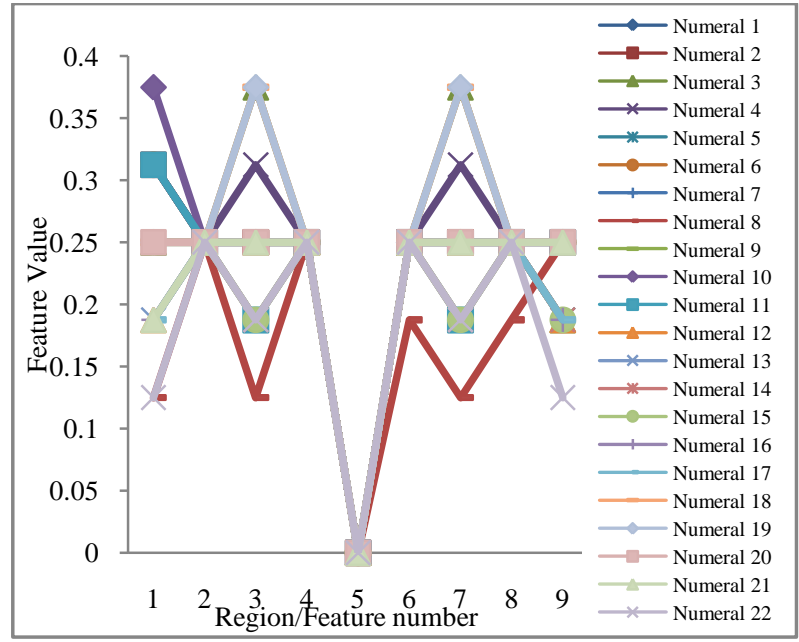

Fig.3. Feature vectors of 22 training samples of 0th numeral class extracted by the approach $M_{1}$

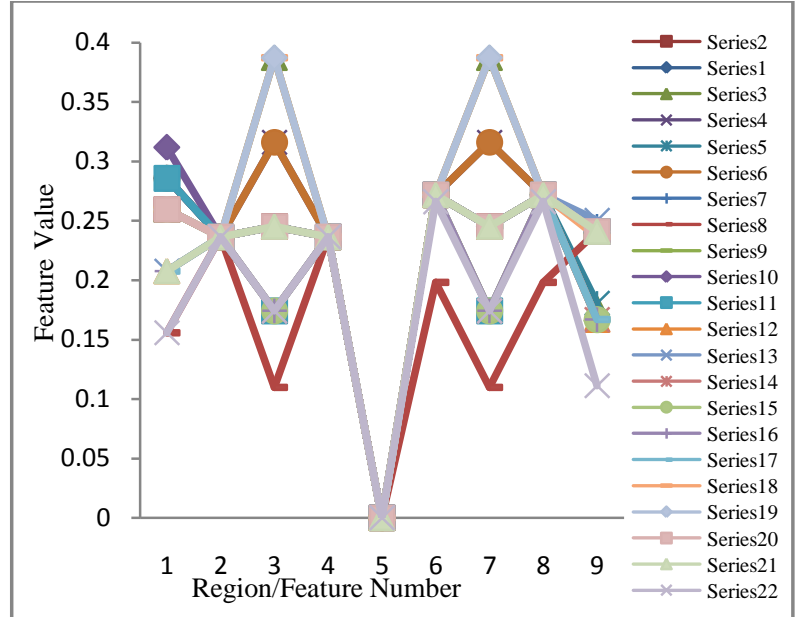

Fig.4. Feature vectors 22 training samples of 0th numeral class extracted by the approach $M_{2}$.

In both the approaches $\mathrm{M}_{1}$ and $\mathrm{M}_{2}$, the feature vectors are overlapped able to see only few lines at most of the regions. This indicates that the feature values have minimum variation among the intraclass in both the approaches $\mathbf{M}_{1}$ and $\mathbf{M}_{2}$. However, it is the four different feature values at region number $1,3,7$ and 9 . This indicates among the twenty-two $0^{\text {th }}$ class samples, only four different styles variation exist. The similar results are found for all other numeral classes. This indicates that the feature extracted by the approaches $\mathrm{M}_{1}$ and $\mathrm{M}_{2}$ are less sensitive to the individual writing style variations.

To know the discriminating ability of extracted features, the mean feature vector of all the numeral classes is computed. Fig.5 and Fig.6 shows the mean feature vectors of the numeral classes computed by the approaches $\mathrm{M}_{1}$ and $\mathrm{M}_{2}$ respectively.

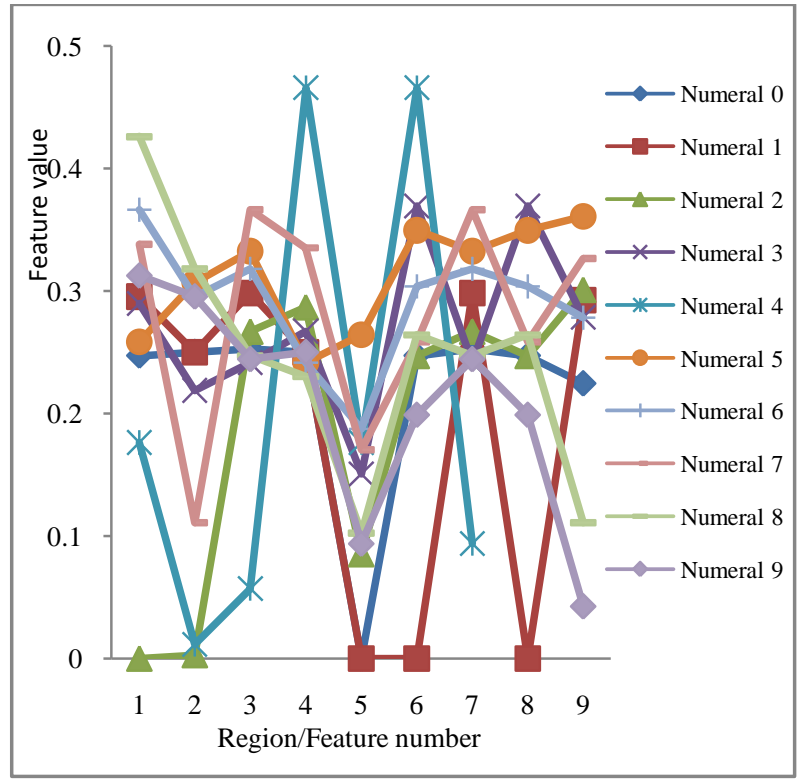

Fig.5. Mean feature vectors of ten numeral classes extracted by the approach $M_{1}$. 


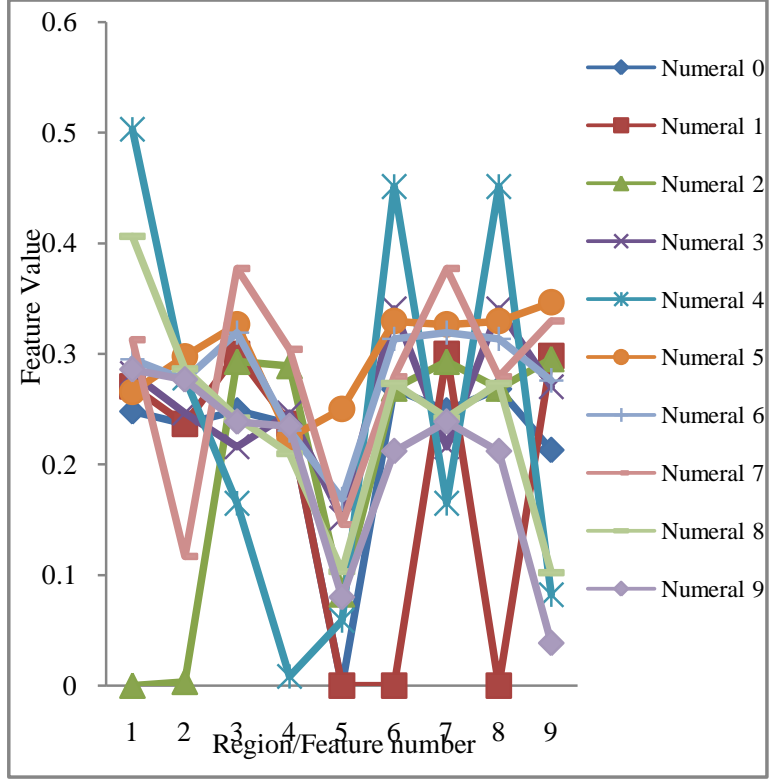

Fig.6. Mean feature vectors of ten numeral classes extracted by the approach $M_{2}$.

It observed that the mean feature vectors are scattered and able to see all the ten vectors at most of the regions numbers. At some regions, the mean features do not differ too much but they have similar characteristics in both the feature extraction approaches for most of the numeral classes. This indicates the extracted feature vectors are distinctive for their class representation.

The feature vectors of individual sample and their numeral classes are shown to the neural network one after the other to learn with different learning rate for both the approaches $\mathrm{M}_{1}$ and $\mathrm{M}_{2}$. Fig. 7 and Fig. 8 shows the number variation in performance indicator RMSE and the number of epochs taken for learning by the approaches $\mathrm{M}_{1}$ and $\mathrm{M}_{2}$ respectively. In the learning process, the same randomly fixed initial hidden and output layer weight matrices are used for both the feature extraction approaches and for all the learning rate values.

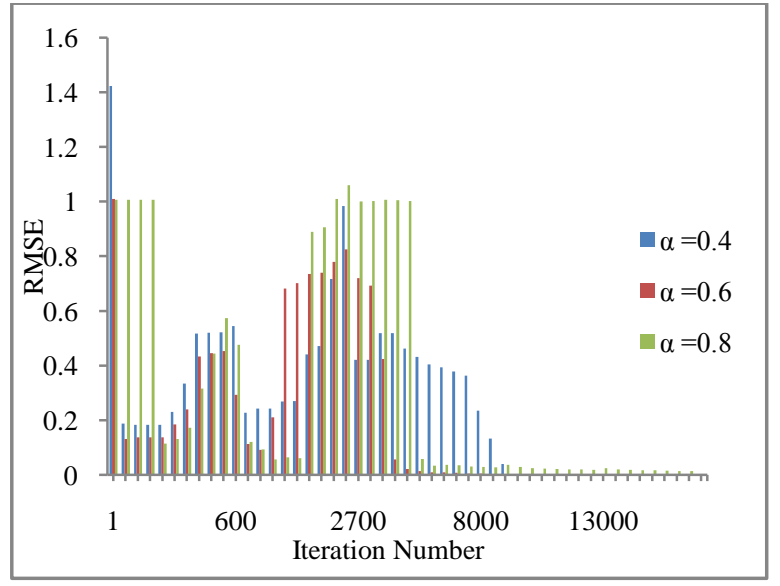

Fig. 7. The variation in performance indicator (RMSE) with the number of epochs while learning for the approaches $M_{1}$.

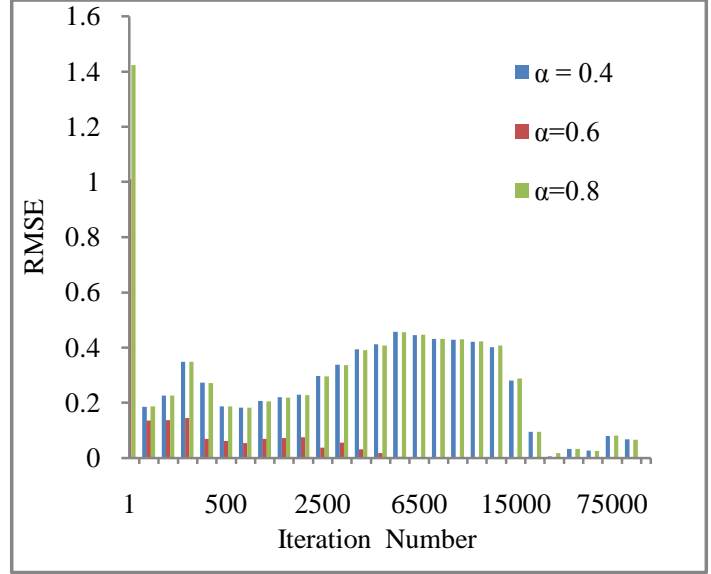

Fig.8. The variation in performance indicator (RMSE) with the number of epochs while learning for the approaches $\mathbf{M}_{2}$.

In both the cases, initially the RMSE values is slightly more than one and it reduces to around 0.2 to 0.3 within few iterations. From the Fig.9, it seen the RMSE value increased momentarily at the initial stage of learning and after some epochs it is reduced in the approach $\mathrm{M}_{1}$ with all the three learning rate values. In the feature extraction approach $\mathrm{M}_{2}$, the RMSE value is not increased to the original value but it took more number of epochs to learn with the learning rate 0.4 and 0.8 . From the Fig.10, it is seen that the FFBPNN the number of epochs taken is less to learn with the learning rate ' $\alpha=$ 0.6. Fig.11 shows the number of epochs taken to lean with the different learning rate for the feature extraction approaches $\mathrm{M}_{1}$ and $\mathrm{M}_{2}$ respectively.

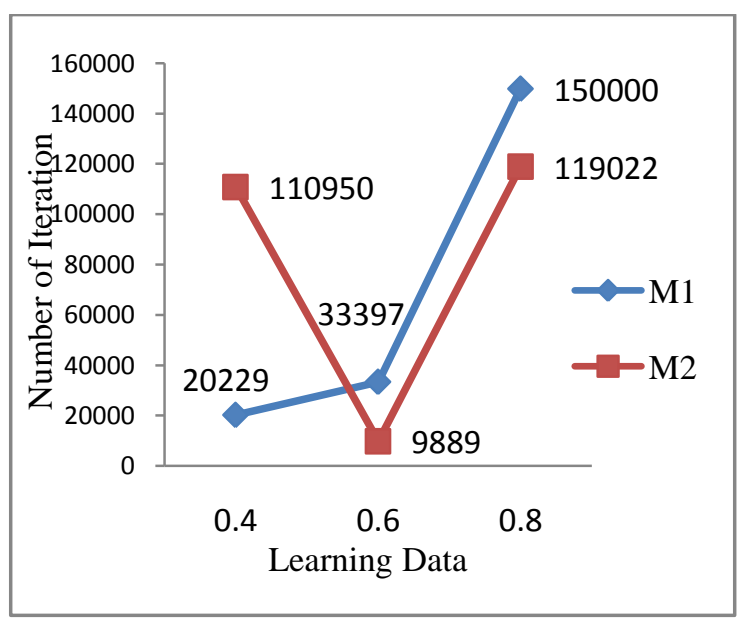

Fig.9. The number of iteration taken to reach the acceptable preset RMSE by the BPNN leaning for the feature extraction approach $M_{1}$ and $M_{2}$.

The FFBPNN took different number of iteration to learn with the RMSE value equal to 0.00015 for different learning rate. It is minimum number of iteration at learning rate $\alpha=0.6$ for the feature extraction approach $\mathbf{M}_{2}$. But, for this approach number of epochs are more when the learning rate value $\alpha=$ 0.4 and 0.8. Comparatively, FFBPNN took minimum number of iteration at $\alpha=0.4$ and 0.6 for learning for the approach $\mathrm{M}_{1}$.

To recognize the unknown samples, 125 Kannada handwritten numeral testing samples feature vectors are shown the trained FFBPNN. The experimental results of feature extraction 
methods $\mathrm{M}_{1}$ and $\mathrm{M}_{2}$ with the different leaning rate is given in Fig.10 and Fig.11 respectively.

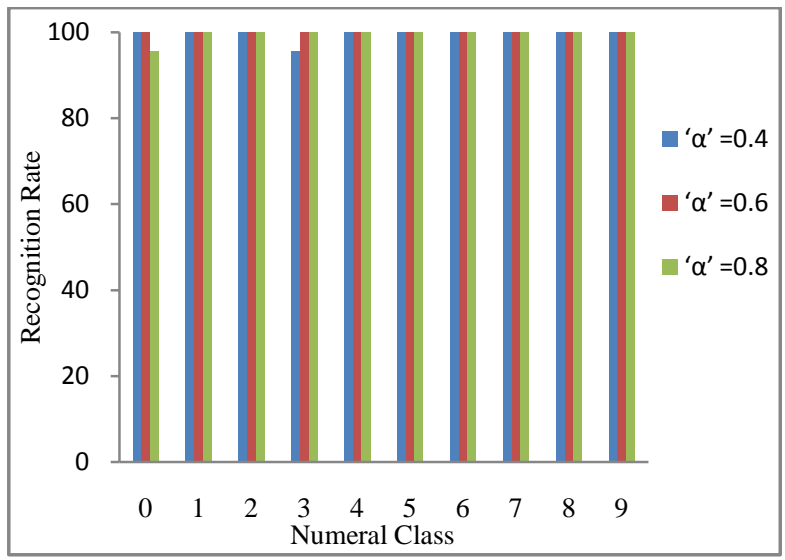

Fig. 10. The recognition rate of Kannada handwritten numeral classes obtained by the $\mathrm{NN}$ and the feature extraction method $M_{1}$.

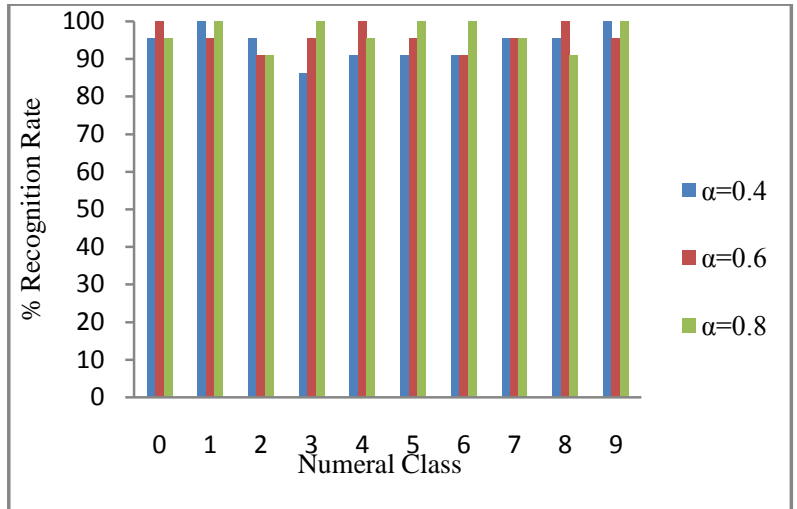

Fig. 11. The recognition rate of Kannada handwritten numeral classes obtained by the $\mathrm{NN}$ and the feature extraction method $\mathrm{M}_{2}$.

The recognition rate of $100 \%$ is obtained for most of the numeral classes for the feature extraction approach $\mathrm{M}_{1}$ and it is slightly less than $100 \%$ for the approach $\mathrm{M}_{2}$. It is found the overall of $99.7 \%$ and $95.5 \%$ is obtained for the feature extraction methods $M_{1}$ and $M_{2}$ respectively.

\section{CONCLUSION}

From the experimental results, following conclusions are drawn. The nine features extracted to represent the Kannada handwritten numerals are sufficient to train the FFBPNN. Feature vectors extracted by the approaches $M_{1}$ and $M_{2}$ are similar characteristics and differ slightly in the feature values. The learning with different leaning rate does not effect too much on the recognition rate but does on the number of epochs. The overall recognition rate of $99.7 \%$ and $95.5 \%$ is obtained for the feature extraction methods $M_{1}$ and $M_{2}$ respectively.

\section{REFERENCES}

[1] Øivind Due Trier, Anil K. Jain and Torfinn Taxt "Feature Extraction Methods for Character Recognition-
A Survey" Pattern Recognition, Vol. 29 No. 4, 1996, pp 641-662.

[2] Nafiz Arica, Fatos T. Yarman-Vural, "An Overview of Character Recognition Focused on Off-line Handwriting", IEEE Transactions on system Man. Cybernetics-Part C: Applications and Reviews, vol. 31 no. 2 2001, pp. 216-233.

[3] Sabri A. Mahmoud "Recognition of writer-independent off-line handwritten Arabic (Indian) numerals using hidden Markov models." Signal Processing, Vol.88, No. 4, 2008, pp - 844-857.

[4] U. Pal, B. B. Chaudhuri, "Indian Script Character recognition: A survey", Pattern Recognition, vol. 37, 2004,pp 1887-1899.

[5] Hanmandlu M., and Murthy O.V.R., "Fuzzy model based recognition of handwritten numerals" Pattern Recognition, vol. 40, 2007,pp 1840 - 1854.

[6] Om Prakash Sharma, M. K. Ghose, Krishna Bikram Shah "An Improved Zone Based Hybrid FeatureExtraction Model for Handwritten Alphabets Recognition Using Euler Number" International Journal of Soft Computing and Engineering, , Vol-2, no.2, May 2012 ,504-508.

[7] Rajashekararadhya S.V. and Vanaja Ranjan P., "Efficient zone based feature extraction algorithm for handwritten numeral recognition of four popular south Indian scripts", Journal of Theoretical and Applied Information Technology, 2005, pp 1171- 1181.

[8] Dinesh Acharya U, N V Subba Reddy and Krishnamoorthi, "Multilevel classifiers in recognition of Handwritten Kannada numerals", World Academy of Science, Engineering and Technology vol.18, 2008, pp278-283.

[9] Dhandra B.V., Gururaj Mukarmbi, and Mallikarjun Hangarge, "A script independent approach for handwritten bi-lingual Kannada and Telugu digits recognition" International Journal of Machine Intelligence, Vol.3 No. 3, 2011, PP- 155 -159.

[10] Umapada Pala, Partha Pratim Royb, Nilamadhaba Tripathya and Josep Lladósb, Multi-oriented Bangla and Devnagari text recognition Pattern Recognition, Vol. 43, No.12, 2010, pp 4124-4136.

[11] Ashoka H.N, Manjaiah D.H, Rabindranath Bera "Feature Extraction Technique for Neural Network Based Pattern Recognition”, International Journal on Computer Science and Engineering (IJCSE), pp 331-339.

[12] Benne R.G., Dhandra B.V. and Mallikarjun Hangarge, "Tri-scripts handwritten numeral recognition: a nove approach" Advances in Computational Research, ISSN 0975-3273, Volume 1, Issue 2, 2009, pp 47-51.

[13] Ashoka H.N, Manjaiah D.H, Rabindranath Bera "Zone Based Feature Extraction and Statistical Classification Technique for Kannada Handwritten Numeral Recognition" International Journal of Computer Science \& Engineering Technology (IJCSET), 476-482. 